\title{
CME Clinical pharmacology (100626): self-assessment
}

\section{questionnaire}

\author{
Edited by Andrew Webb and Tahseen A Chowdhury
}

\section{SAQs and answers are ONLINE for RCP fellows and collegiate members}

SAQs and answers are ONLINE for RCP fellows and collegiate members

\section{Format}

Candidates are asked to choose the best answer from the five possible answers. This best of five format is used in many medical examinations, however the questions are not intended to be representative of those used in the MRCP(UK) Part 1 or Part 2 Written Examinations.

\section{The answering process}

1 Go to www.rcplondon.ac.uk/SAQ

2 Log on using your usual RCP username and password

3 Select the relevant CME question paper

4 Answer all 10 questions by selecting the best answer from the options provided

5 Once you have answered all the questions, click on Submit

\section{Registering your external CPD credits}

Carrying out this activity allows you to claim two external CPD credits. These will be automatically transferred to your CPD diary, where you can review the activity and claim your points.

1 A 75-year-old woman was reviewed in a general medical clinic. She was known to be hypertensive, had atrial fibrillation and chronic kidney disease stage 3. Her medication included aspirin, bendroflumethiazide, lisinopril, digoxin and atorvastatin.

On examination her heart rate was $56 \mathrm{bpm}$, irregular and the average of three blood pressure measurements was $162 / 82 \mathrm{mmHg}$.

What is the most appropriate further medicine to control her hypertension?
(a) amlodipine
(b) bisoprolol
(c) doxazosin
(d) spironolactone
(e) verapamil.

2 A 47-year-old woman with alcohol dependence was admitted with a 3-day history of increasing abdominal pain and nausea. Her medications were amlodipine $10 \mathrm{mg}$ orally daily and thiamine $50 \mathrm{mg}$ orally daily. She admitted to drinking around one bottle of vodka per day but denied any intake over the preceding 3 days.

On examination she was apyrexial, had a regular heart rate of $100 \mathrm{bpm}$ and blood pressure $120 / 84 \mathrm{mmHg}$. She was jaundiced, irritable and had a marked tremor of the hands. Abdominal examination revealed $4 \mathrm{~cm}$ tender hepatomegaly with no clinical evidence of ascites. Her memory was poor and she was disorientated in time and place.

\section{Investigations}

$\mathrm{Na}+131 \mathrm{mmol} / \mathrm{L}$ (137-144), K+ $3.6 \mathrm{mmol} / \mathrm{L}$ (3.5-4.9), U $2.1 \mathrm{mmol} / \mathrm{L}$ (2.5-7.0), Cr $56 \mu \mathrm{mol} / \mathrm{L}$ (60-110), plasma glucose $5.3 \mathrm{mmol} / \mathrm{L}$, albumin $26 \mathrm{~g} / \mathrm{L}$ (37-49), bilirubin 80 $\mu \mathrm{mol} / \mathrm{L}$ (1-22), ALT $345 \mathrm{U} / \mathrm{L}$ (5-35), ALP $164 \mathrm{U} / \mathrm{L}$ (45-105).

Which of the following options would be the best treatment at this stage?
(a) enoxaparin $40 \mathrm{mg}$ (4000 units) SC daily
(b) glucose $5 \% 500 \mathrm{~mL}$ with $10 \mathrm{mmol} \mathrm{KCl} \mathrm{IV} \mathrm{over} 4$ hours
(c) lorazepam $1 \mathrm{mg}$ IV over 2 minutes
(d) pabrinex® I/V High Potency by IV infusion over 30 minutes
(e) sodium chloride $0.9 \% 1 \mathrm{~L}$ with $20 \mathrm{mmol} \mathrm{KCl} \mathrm{IV} \mathrm{over} 2$ hours.

3 Which of the following is considered an implicit indicator of potentially inappropriate prescribing?
(a) any duplicate drug class prescription
(b) COX-2 selective NSAIDs with concurrent cardiovascular disease
(c) digoxin for heart failure with preserved systolic ventricular function
(d) levodopa or dopamine agonists for benign essential tremor
(e) ticlopidine in any circumstances.

4 Which of the following medications is most commonly prescribed in UK primary care?
(a) antimicrobials
(b) cardiovascular
(c) gastrointestinal
(d) musculoskeletal
(e) skin. 
5 Which four groups of medicines are linked to around $50 \%$ of preventable drug-related admissions according to Howard et al?

(a) antiplatelets, anticoagulants, insulin and nonsteroidal antiinflammatory drugs (NSAIDs)

(b) antiplatelets, diuretics, nonsteroidal anti-inflammatory drugs (NSAIDs) and anticoagulants

(c) insulin, anticoagulants, monoclonal antibodies and antiplatelets

(d) nonsteroidal anti-inflammatory drugs (NSAIDs), antiplatelets, insulin and anticoagulants

(e) nonsteroidal anti-inflammatory drugs (NSAIDs), monoclonal antibodies, anticoagulants and diuretics.

6 According to Barber et al, what percentage of patients are non-adherent to their new medicines 10 days after starting them?
(a) $10 \%$
(b) $22 \%$
(c) $34 \%$
(d) $43 \%$
(e) $55 \%$

7 In general, angiotensin converting enzyme (ACE) inhibitors are preferred to angiotensin receptor blockers (ARBs) in the treatment of heart failure. Why, then, is the recently approved neprilysin inhibitor sacubitril combined with the ARB valsartan?
(a) better efficacy
(b) better pharmaco-economics
(c) ease of administration
(d) less frequently dosed
(e) less severe side effects, including angioedema.

8 How many of the 91 medicines approved in 2015 are characterised by a novel mechanism of action?
(a) $4 \%$
(b) $16 \%$
(c) $25 \%$
(d) $33 \%$
(e) $36 \%$

9 Which one of the following statements is correct regarding reports to the Yellow Card Scheme?

(a) A black triangle identifies new drugs that are being intensively monitored by regulators (b) A suspected adverse drug reaction (ADR) as a result of a licensed medicine can be reported to the scheme, and those to unlicensed medicines reported directly to the manufacturer

(c) All suspected reactions to all drugs should be reported to the scheme

(d) Reports for ADRs associated with herbal medicines and vaccines are exempt from the scheme

(e) The reporter of the ADR must be a registered healthcare practitioner.

10 A 55-year-old man of African-Caribbean race was seen in a hypertension clinic. He had been on therapy with felodipine $2.5 \mathrm{mg}$ daily and enalapril $10 \mathrm{mg}$ daily for several years. A week before the appointment he suffered an episode of severe lip swelling after a routine dental appointment. The swelling disappeared after a few hours and the patient assumed that it was a reaction to one of the dental products. He refused to go to the emergency department even though he told his wife that he felt as though he was choking. The clinic doctor suspects that the patient is describing an episode of ACE inhibitor-induced angioedema.

\section{Which of the following statements is true?}

(a) As the angioedema has resolved, no change in antihypertensive treatment is needed

(b) The adverse effect has resolved despite continuing the drug therefore the angioedema is unlikely to be drug induced

(c) The adverse effect is unlikely to have been caused by the enalapril as the patient has been on treatment for several years

(d) The clinic doctor should report the ADR on a Yellow Card based on the suspicion of a serious reaction

(e) The patient's ethnicity has no significance to the probability of this being an ADR.

\section{CME Movement disorders SAQ}

Answers to the CME SAQ published in Clinical Medicine in August 2016

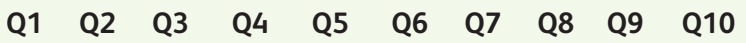
(e)
(b) (c)
(b)
(c)
(e)
(b) (b)
(c) 